\title{
Compressive Deformation Behavior at Elevated Temperatures in a Closed-Cell Aluminum Foam
}

\author{
Masataka Hakamada, ${ }^{1, *}$, Tatsuho Nomura ${ }^{1, *}$, Yasuo Yamada ${ }^{2}$, Yasumasa Chino ${ }^{2}$, \\ Youqing Chen ${ }^{1}$, Hiromu Kusuda ${ }^{1}$ and Mamoru Mabuchi ${ }^{1}$ \\ ${ }^{1}$ Department of Energy Science and Technology, Graduate School of Energy Science, Kyoto University, Kyoto 606-8501, Japan \\ ${ }^{2}$ Materials Research Institute for Sustainable Development, National Institute of Advanced Industrial Science and Technology, \\ Nagoya 463-8560, Japan
}

\begin{abstract}
Compressive tests at temperatures of $573-773 \mathrm{~K}$ with initial strain rates of $8.0 \times 10^{-4}-2.0 \times 10^{-1} \mathrm{~s}^{-1}$ were carried out on a closed-cell aluminum foam and its bulk reference aluminum (a cell wall material of the aluminum foam). As a result, the stress exponent and the activation energy for elevated temperature deformation of the aluminum foam were in agreement with those of the bulk reference aluminum. In addition, upon compensation by the relative density, the plateau stress of the aluminum foam was comparable to the stress of the bulk reference aluminum. It is concluded that the elevated temperature deformation mechanism in the aluminum foam is essentially the same as that in the bulk reference aluminum.
\end{abstract}

(Received March 30, 2005; Accepted May 19, 2005; Published July 15, 2005)

Keywords: aluminum, foam, cellular solid, elevated temperature deformation, mechanical properties, compressive test

\section{Introduction}

Aluminum foam is one of ultralight materials exhibiting many unique characteristics in mechanical, thermal, electrical, and acoustic properties. ${ }^{1,2)}$ Mechanical properties at room temperature have been studied extensively for metallic foams. ${ }^{1)}$ However, their mechanical properties at elevated temperatures have been much less documented. Recently, Andrews et al. ${ }^{3)}$ developed the expression for creep bending of cell walls in a metallic foam. According to their results, the strain rate for a metallic foam is given by

$$
\begin{aligned}
& \frac{\dot{\varepsilon}^{*}}{\dot{\varepsilon}_{0}}=\left[\frac{\sigma^{*} / \sigma_{0}}{\frac{1}{1.7}\left(\frac{n+2}{0.6}\right)^{1 / n}\left(\frac{n}{2 n+1}\right)\left(\phi \frac{\rho^{*}}{\rho}\right) \frac{3 n+1}{2 n}+\frac{2}{3}(1-\phi) \frac{\rho^{*}}{\rho}}\right]^{n} \\
& \dot{\varepsilon}_{0}=A \exp \left(-\frac{Q}{R T}\right),
\end{aligned}
$$

where $\dot{\varepsilon}^{*}$ is the strain rate for a metallic foam, $\sigma^{*}$ is the applied stress, $\rho^{*}$ is the density of the metallic foam, $\rho$ is the density of a bulk reference metal of which the cell walls are made, $\phi$ is the volume fraction of solid in the cell edges, $n$ is the stress exponent, $\dot{\varepsilon}_{0}$ and $\sigma_{0}$ are constants related to the bulk reference metal, $A$ is a constant, $Q$ is the activation energy for deformation at elevated temperature and $T$ is the absolute temperature. According to eqs. (1) and (2), the stress exponent for a metallic foam is expected to agree with that for the bulk reference metal. In general, the stress exponent for a dislocation creep region is $4-6$ for a metallic solid. However, several experimental results revealed that the stress exponents for aluminum foams are 12-15 and these values are much larger than expected for a metallic solid. ${ }^{4-6)}$ This suggests that there should be difference in stress exponents, which characterizes deformation at elevated temperatures, between the metallic foams and the metallic solid. However, there have been few investigations that compare elevated temperature compressive properties of metallic foams with those of their bulk reference metals.

\footnotetext{
*Graduate student, Kyoto University.
}

In the present study, compressive tests at elevated temperatures with various strain rates were carried out on a closedcell aluminum foam and its bulk reference aluminum with the same chemical composition as the aluminum foam, to compare elevated temperature compressive properties in the aluminum foam with those in the bulk reference aluminum.

\section{Experimental}

The material subjected to the compressive tests was a commercially available closed-cell aluminum foam (trade "ALPORAS", Shinko Wire Company Ltd., Amagasaki, Japan). The relative density of the aluminum foam sample was $10 \%$. The detail of fabrication process for the aluminum foam has been described elsewhere. ${ }^{7}$ The specimens with the dimensions of $20 \mathrm{~mm} \times 20 \mathrm{~mm} \times 22 \mathrm{~mm}$ were cut from a purchased block for compressive tests. For comparison, a bulk reference aluminum, whose chemical compositions were the same as those of the aluminum foam, were produced by melting the foam in a high frequency induction furnace under an argon atmosphere. The dimensions of specimens for 
(a)

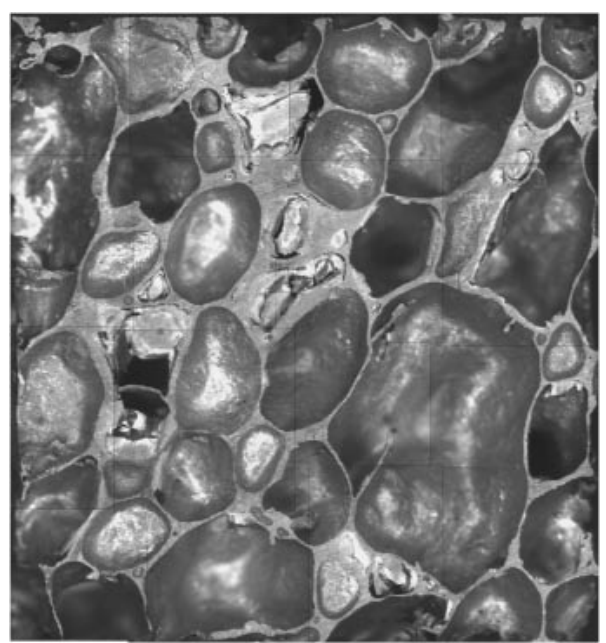

(b)

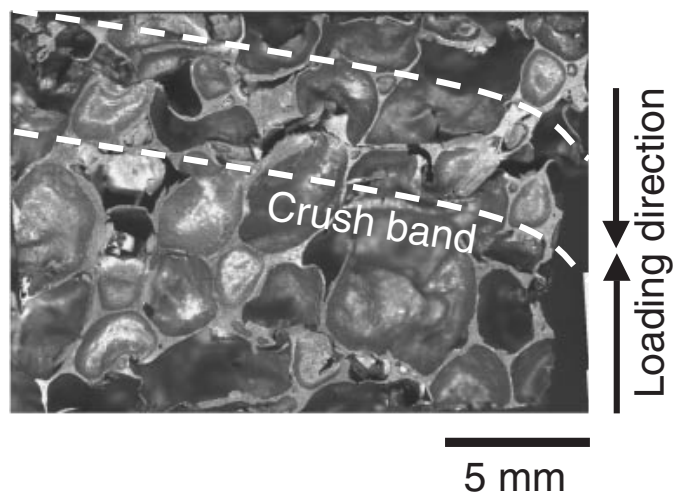

Fig. 1 Surface of the aluminum foam: (a) before testing and (b) after deformation at $\varepsilon=32 \%$ at room temperature.

the bulk reference aluminum were $5 \mathrm{~mm} \times 5 \mathrm{~mm} \times 7 \mathrm{~mm}$.

Compressive tests were carried out at room temperature, 573,673 and $773 \mathrm{~K}$ with initial strain rates of $8.0 \times 10^{-4}$, $6.0 \times 10^{-3}, 4.0 \times 10^{-2}$ and $2.0 \times 10^{-1} \mathrm{~s}^{-1}$. The deviation in testing temperature was within $1 \mathrm{~K}$. The surfaces of the specimens deformed at room temperature and $573 \mathrm{~K}$ were observed under an optical microscope to investigate the collapse mechanism.

\section{Results}

Figure 1 shows the surface of the aluminum foam before deformation and after deformation at $\varepsilon=32 \%$ at room temperature. Deformation was localized and a crush band was found. This trend is the same as those in the previous works. $^{1,2)}$ Figure 2 shows the surface of the aluminum foam before and after deformation at $\varepsilon=30 \%$ at $573 \mathrm{~K}$ with $4.0 \times 10^{-2} \mathrm{~s}^{-1}$. A crush band was locally found at $573 \mathrm{~K}$ as well as at room temperature, indicating that the collapse mechanism at elevated temperatures is similar to that at room temperature in the aluminum foam.

The nominal stress-nominal strain curves at $673 \mathrm{~K}$ with $8.0 \times 10^{-4}-2.0 \times 10^{-1} \mathrm{~s}^{-1}$ for the aluminum foam are shown in Fig. 3. The curves exhibited typical deformation characteristics of foamed materials, that is, an elastic region in an initial stage of deformation, then a plateau region with a (a)

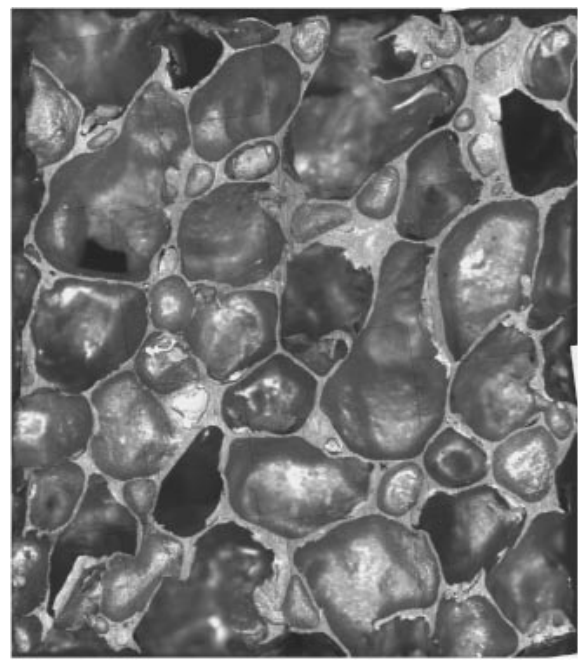

(b)

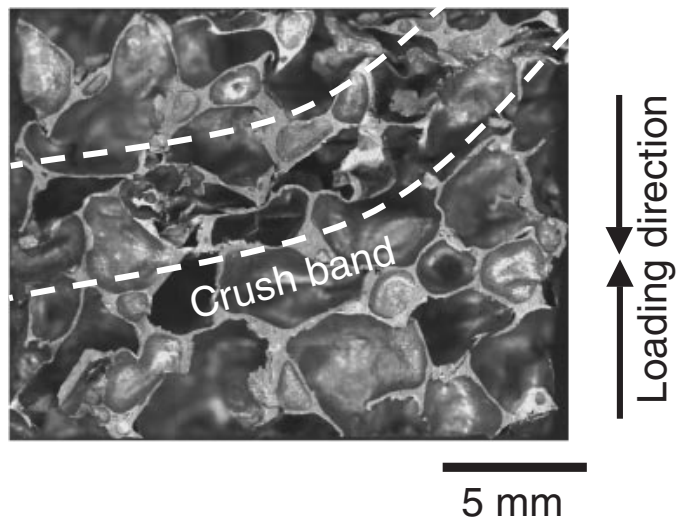

Fig. 2 Surface of the aluminum foam: (a) before testing and (b) after deformation at $\varepsilon=30 \%$ at $573 \mathrm{~K}$ with an initial strain rate of $4.0 \times$ $10^{-2} \mathrm{~s}^{-1}$.

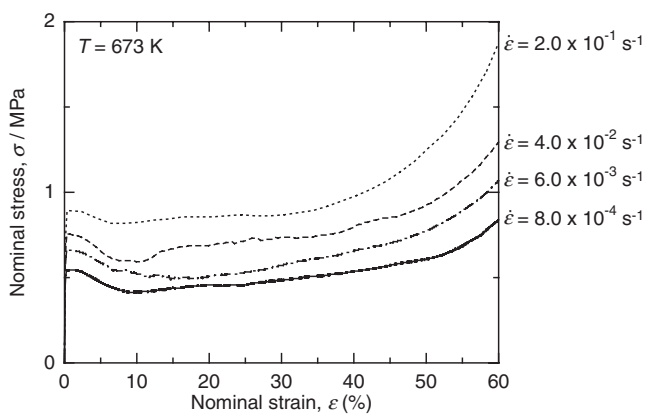

Fig. 3 Nominal stress-nominal strain curves at $673 \mathrm{~K}$ with initial strain rates of $8.0 \times 10^{-4}-2.0 \times 10^{-1} \mathrm{~s}^{-1}$ for the aluminum foam.

nearly constant stress over a wide range of strain and finally a densification region where the stress increases rapidly. Thus, these three distinct deformation regions were observed at elevated temperatures as well as at room temperature. This corresponds to the fact that the collapse mechanism at elevated temperatures is similar to that at room temperature in the aluminum foam.

The true stress-true strain curves at $673 \mathrm{~K}$ with $8.0 \times$ $10^{-4}-2.0 \times 10^{-1} \mathrm{~s}^{-1}$ for the bulk reference aluminum are 


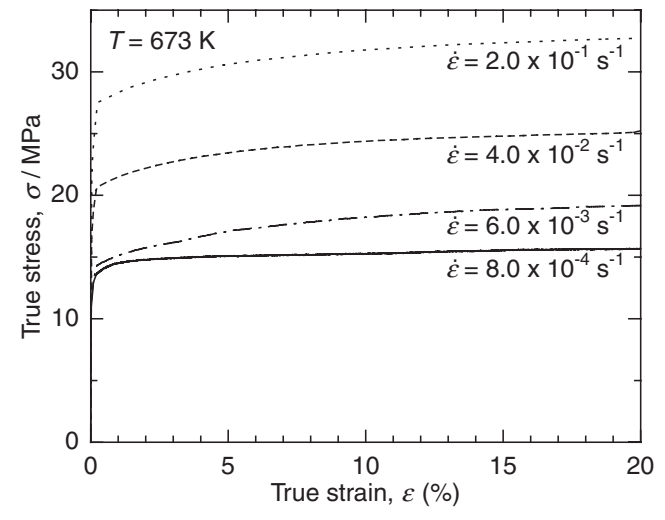

Fig. 4 True stress-true strain curves at $673 \mathrm{~K}$ with initial strain rates of $8.0 \times 10^{-4}-2.0 \times 10^{-1} \mathrm{~s}^{-1}$ for the bulk reference aluminum.

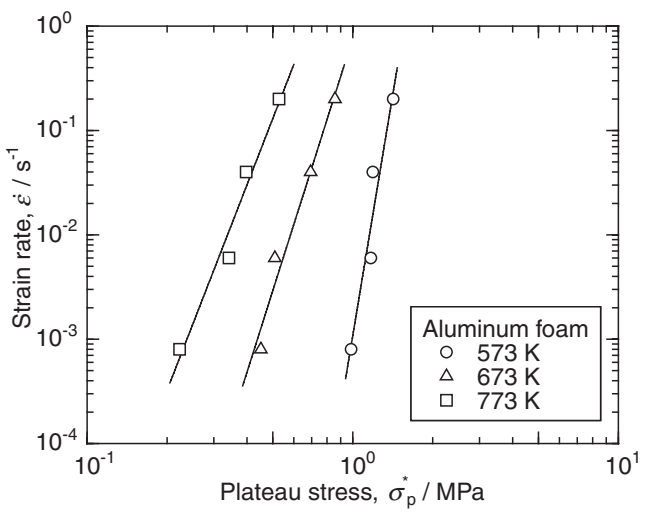

Fig. 5 Relationship between the plateau stress and the strain rate at 573$773 \mathrm{~K}$ for the aluminum foam.

shown in Fig. 4. The flow stress became constant soon after yielding or slight strain hardening under all the investigated testing conditions.

\section{Discussion}

\subsection{Stress/strain relationship}

The relationship between the stress and the strain rate at 573-773 K for the aluminum foam is shown in Fig. 5, where the stress of the aluminum foam is the plateau stress which is defined as the average flow stress in the range of $\varepsilon=$ $15-25 \%$. The values of stress exponent were 15.3 at $573 \mathrm{~K}$, 8.0 at $673 \mathrm{~K}$ and 6.5 at $773 \mathrm{~K}$. The stress exponent value at $573 \mathrm{~K}$ was much larger than those at 673 and $773 \mathrm{~K}$.

The relationship between the stress and the strain rate at $573-773 \mathrm{~K}$ for the bulk reference aluminum is shown in Fig. 6, where the stress of the bulk reference aluminum is the true stress under a steady state. The stress exponent value was 11.0 at $573 \mathrm{~K}, 6.4$ at $673 \mathrm{~K}$ and 7.1 at $773 \mathrm{~K}$. It should be noted that the trend of the larger stress exponent at $573 \mathrm{~K}$ for the bulk reference aluminum is the same as that for the aluminum foam and that the stress exponent value for the aluminum foam is comparable to that for the bulk reference aluminum at each testing temperature. The larger stress exponent at $573 \mathrm{~K}$ is likely to be attributed to power-law breakdown behavior.

The variation in strain rate as a function of reciprocal

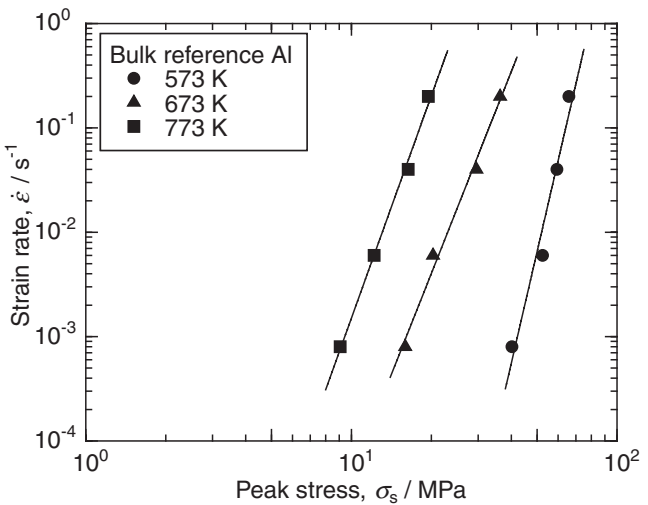

Fig. 6 Relationship between the peak stress and the strain rate at 573$773 \mathrm{~K}$ for the bulk reference aluminum.

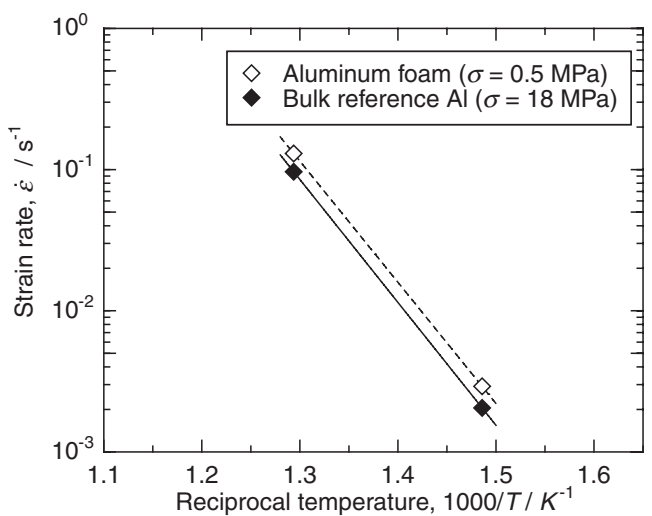

Fig. 7 Variation in strain rate as a function of reciprocal temperature for the aluminum foam and the bulk reference aluminum.

temperature for the aluminum foam and its bulk reference aluminum is shown in Fig. 7, where each stain rate is estimated at a constant stress of $0.5 \mathrm{MPa}$ for the aluminum foam and at $18 \mathrm{MPa}$ for the bulk reference aluminum. From the slopes of the plots in Fig. 7, the activation energies for deformation at elevated temperatures were calculated to be $164 \mathrm{~kJ} \mathrm{~mol}^{-1}$ in the aluminum foam and $167 \mathrm{~kJ} \mathrm{~mol}^{-1}$ in the bulk reference aluminum. It is of interest to note that the activation energy for deformation at elevated temperatures in the aluminum foam is in agreement with that in the bulk reference aluminum and these values are comparable to the activation energy for lattice diffusion of aluminum. Thus, the experimental results showed that the stress exponent and activation energy in the aluminum foam are comparable to those in the bulk reference aluminum. This indicates that the deformation mechanism of the aluminum foam is the same as that of the bulk reference aluminum.

\subsection{Compensation by relative density}

Gibson and Ashby ${ }^{1)}$ developed the relationship between the relative stress $\left(=\sigma_{\mathrm{p}}^{*} / \sigma_{\mathrm{ys}}\right)$ and the relative density $\left(=\rho^{*} / \rho_{\mathrm{s}}\right)$. According to their works, the relationship is given by

$$
\frac{\sigma^{*} \mathrm{p}}{\sigma_{\mathrm{ys}}}=C\left(\frac{\rho^{*}}{\rho_{\mathrm{s}}}\right)^{3 / 2},
$$




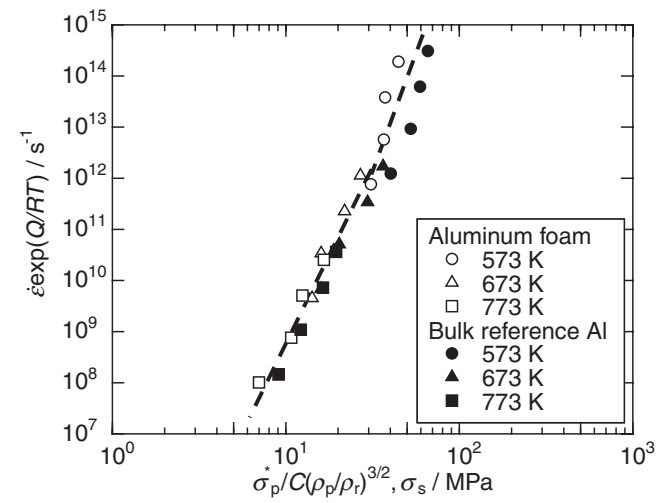

Fig. 8 Relationship between the compensated plateau stress of the aluminum foam $\left(=\sigma^{*}{ }_{\mathrm{p}} / C\left(\rho^{*} / \rho_{\mathrm{s}}\right)^{3 / 2}\right)$ or the peak stress of the bulk reference aluminum $\left(=\sigma_{\mathrm{s}}\right)$ and $\dot{\varepsilon} \exp (Q / R T)$, where $C$ is 1.07 . See the text for detailed information on symbols in the expressions.

where $\sigma^{*}$ is the plateau stress of a foam, $\sigma_{\mathrm{ys}}$ is the yield stress of a bulk reference material, $\rho^{*}$ is the density of the foam, $\rho_{\mathrm{s}}$ is the density of the bulk reference material and $C$ is a constant. From eq. (3), the stress in the plateau region of the aluminum foam may be compensated by the relative density and the constant $C$. The compensated plateau stress of the aluminum foam is then represented as $\sigma^{*}{ }_{\mathrm{p}} / C\left(\rho^{*} / \rho_{\mathrm{s}}\right)^{3 / 2}$. The value of $C$ for the aluminum foam was calculated to be 1.07 from the results of the compressive test at room temperature. As shown in Figs. 1 and 2, the collapse mechanism at elevated temperatures was the same as that at room temperature. Hence, the stress in the plateau region at elevated temperatures may be compensated by the value of $C$ at room temperature.

The relationship between the compensated plateau stress of the aluminum foam, that is, $\sigma^{*}{ }_{\mathrm{p}} / C\left(\rho^{*} / \rho_{\mathrm{s}}\right)^{3 / 2}$, or the peak stress of the bulk reference aluminum $\left(=\sigma_{\mathrm{s}}\right)$ and $\dot{\varepsilon} \exp (Q / R T)$ is shown in Fig. 8, where $\dot{\varepsilon}$ is the testing strain rate, $Q$ is the activation energy, $R$ is the gas constant and $T$ is the testing absolute temperature. It can be seen that the plateau stress of the aluminum foam is successfully compensated to the peak stress of the bulk reference aluminum by the relative density. Therefore, it is concluded that there is no essential difference in deformation mechanism between the aluminum foam and its bulk reference aluminum.

\section{Summary}

Compressive tests at $573-773 \mathrm{~K}$ were carried out on a closed-cell aluminum foam and its bulk reference aluminum to compare compressive properties of the aluminum foam with those of the bulk reference aluminum.

The observation of the deformed specimens revealed that the collapse mechanism at elevated temperatures is the same as that at room temperature, which coincides with the fact that the plateau region in the stress-strain curves was found at elevated temperatures as well as at room temperature.

The stress exponent and activation energy for deformation at elevated temperatures in the aluminum foam were roughly in agreement with those in the bulk reference aluminum. In addition, upon compensation by the relative density, the plateau stress of the aluminum foam was comparable to the stress of the bulk reference aluminum. Therefore, it is concluded that the deformation mechanism at elevated temperatures in the aluminum foam is essentially the same as that in the bulk reference aluminum.

\section{Acknowledgement}

The authors thank to the 21st Century COE Program "Establishment of COE on Sustainable Energy System" (Ministry of Education, Culture, Sports, Science and Technology) for financial support.

\section{REFERENCES}

1) L. J. Gibson and M. F. Ashby: Cellular Solids: Structure and Properties, (Pergamon Press, Oxford, 1988).

2) J. Banhart: Prog. Mater. Sci. 46 (2001) 559-632.

3) E. W. Andrews, L. J. Gibson and M. F. Ashby: Acta Mater. 47 (1999) 2853-2863.

4) E. W. Andrews, J.-S. Huang and L. J. Gibson: Acta Mater. 47 (1999) 2927-2935.

5) P. Zhang, M. Haag, O. Kraft, A. Wanner and E. Arzt: Philos. Mag. A 82 (2002) 2895-2907.

6) M. Haag, A. Wanner, H. Clemens, P. Zhang, O. Kraft and E. Arzt: Metall. Mater. Trans. A 34A (2003) 2809-2817.

7) T. Miyoshi, M. Itoh, S. Akiyama and A. Kitahara: Adv. Eng. Mater. 2 (2000) 179-183. 